\title{
Basilar Artery Embolism Mimicking Transient Global Amnesia in a 61-Year-Old Female: A Case Report
}

\author{
Ernst Mayerhofer ${ }^{a}$ b Dirk Kanz $^{a} \quad$ Tuba Avcil $^{c} \quad$ Christian Taschner ${ }^{c}$ \\ Christoph Strecker $^{a} \quad$ Wolf-Dirk Niesen ${ }^{a}$ \\ ${ }^{a}$ Clinic of Neurology and Neurophysiology, Medical Center - University of Freiburg, \\ Faculty of Medicine, University of Freiburg, Freiburg, Germany; ${ }^{b}$ Department of Neurology, \\ Massachusetts General Hospital, Harvard Medical School, Boston, MA, USA; 'Clinic of \\ Neuroradiology, Medical Center - University of Freiburg, Faculty of Medicine, University of \\ Freiburg, Freiburg, Germany
}

\author{
Keywords \\ Cerebrovascular ischemia - Transient global amnesia - CNS diseases · Dissection · Duplex \\ sonography · Doppler sonography
}

\section{Abstract}

Transient global amnesia (TGA) is a self-limiting neurological condition that temporarily affects patients' ability to access and store memories. So far, its etiology is unknown; however, ischemic origin has been discussed in the past. We present the case of a 61-year-old female with clinical appearance of TGA. MRI and duplex scan revealed punctiform and patchy ischemic lesions in both temporal lobes and right vertebral artery dissection, suggesting basilar artery embolism as the underlying cause. Our case report shows that TGA can be a symptom of ischemic lesions in the hippocampus and patients with presentation of additional focal neurologic symptoms or atypical distribution or appearance of the diffusion-weighted image (multiple/patchy) lesions should get ischemic stroke diagnosis and treatment. 
Mayerhofer et al.: Embolism Mimicking Transient Global Amnesia

\section{Introduction}

Transient global amnesia (TGA) is a neurological syndrome with acute onset of anterograde memory loss and inability to learn newly acquired information without other neurological deficits, which resolves spontaneously within $24 \mathrm{~h}[1,2]$. Patients often retain a circumscriptive memory loss for the time of the attack. The etiology is unknown, although venous, vascular, migrainous, epileptic, and psychogenic mechanisms are discussed [3]. Evidence of MRI imaging in TGA patients is conflicting: frequently, but not always, punctiform diffusion restrictions and sometimes hyperintensities on T2-weighted sequences on either or even both hippocampi are found within $48 \mathrm{~h}$ of symptom onset [4-7]. Especially and characteristically in studies where MRI was performed within the first $24 \mathrm{~h}$ of onset, no abnormal patterns were seen $[8,9]$. Despite the characteristic hippocampal diffusion restrictions, embolic ischemic origin has not been proven so far but is suspected indirectly via a higher incidence of patent foramen ovale in TGA. There are only few descriptions of proven ischemic etiology, such as in one particular case where TGA-like clinical presentation was found as a result of an embolic shower in the posterior circulation after cerebral angiography [10]. Larger isolated hippocampal infarcts usually present with dizziness, confusion, cognitive or memory deficits, or altered consciousness [11]. Although an ischemic origin is discussed, the risk of cerebral ischemia is not elevated in patients with TGA compared to such with transient ischemic attack [12]. In this report, we discuss a case of clinical presentation of TGA accompanied by mild symptoms of vertebrobasilar ischemia, leading to the diagnosis of vertebral artery dissection with possible basilar artery embolism.

\section{Case Presentation}

A61-year-old female patient developed a sudden onset of general weakness and dizziness while dying her hair. Her daughter also observed memory loss in the patient and called emergency services. On admission, the patient was conscious, partially oriented, and showed amnesia for the current day and the day before while repeatedly asking stereotypical questions. The neurological exam was unremarkable except for a doubtful subtle hemiataxia on the left side. Native brain CT scan was normal. CT-angiography was performed to rule out vertebral or basilar artery occlusion and showed a 9-mm aneurysm at the origin of the right vertebral artery as well as caliber irregularities over the whole length of the hypoplastic (but in basilar artery ending) vessel, suspicious for chronic vertebral artery dissection. Duplex scan revealed a high-grade stenosis at the origin of the right vertebral artery with a dissecting aneurysm and intramural hematoma in the proximal segment of the vessel (Fig. 1). In suspicion of transient basilar artery embolism, $300 \mathrm{mg}$ acetylsalicylic acid (ASA) was administered. Thrombolysis was not performed because of the unusual presentation, uncertainty of the ischemic cause, and only minor neurological deficit summing up to a low NIH-SS of 2. On the following day, neurological exam was normal, and memory capacity was restored except for a memory gap of 3-4 h surrounding the symptom onset. A brain MRI (1.5 T) performed $25 \mathrm{~h}$ after symptom onset revealed multiple small diffusion-weighted images (DWI) hyperintensities with corresponding apparent diffusion coefficient lesions in both hippocampi (Fig. 2). Besides above-mentioned vascular findings, clinical workup showed hypercholesterolemia, mild cervical atherosclerosis, history of hypertension, chronic venous insufficiency, and benign paroxysmal positional vertigo. The patient did not have a history of seizures and the electroencephalogram was unremarkable. We did not rule out vasculitis because there were no other ischemic lesions on DWI/FLAIR and no other artery abnormalities. Because of the intracranial portion of the dissection, we decided against oral anticoagulation

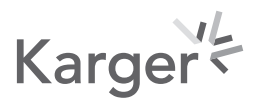


Fig. 1. Duplex scan revealed thrombus formation within the aneurysm at the origin of the $\mathrm{R}-\mathrm{VA}$, resulting in high-grade stenosis. R-SUBCLAV, right subclavian artery; R-VA, right vertebral artery.

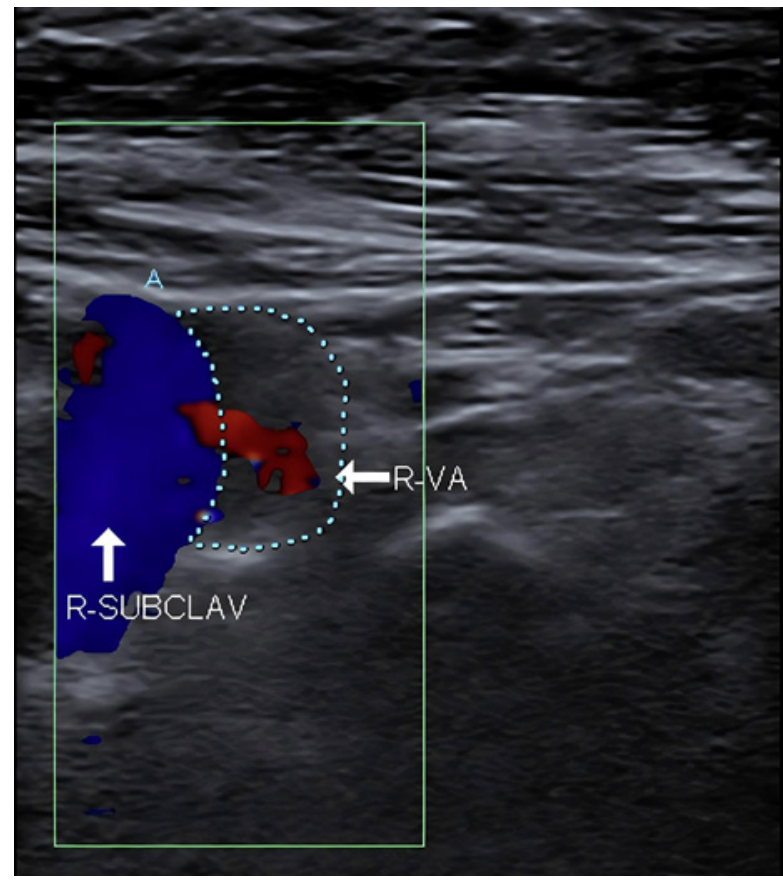

and continued the antithrombotic secondary prevention with ASA $100 \mathrm{mg}$, clopidogrel $75 \mathrm{mg}$, and atorvastatin $80 \mathrm{mg}$ daily. The patient was discharged with persistent amnesia for the initial event but no other signs or symptoms. Duplex scan after 2 months showed progression of the stenosis now with thrombotic occlusion of the aneurysm at the origin of the right vertebral artery and retrograde flow in the intracranial part of the vessel. Therefore, we continued the dual antithrombotic regimen with ASA/clopidogrel for another 6 weeks. After 6 months, the patient experienced sudden onset of headache and dizziness with numbness of the left arm lasting for a few minutes. On admission, she reported headache but had no neurological signs. CT-angiography and MRI showed stable findings of the vessels. She was discharged without symptoms apart from still persistent amnesia for the first event.

\section{Discussion}

Despite amnesia being the major symptom, our patient did not fulfill the TGA criteria proposed by Hodges and Warlow [1] due to the additional neurological deficits hemiataxia, dizziness, and general weakness. For that reason, vessel imaging in suspicion of acute ischemic stroke was performed. The manipulation of the head and neck while dying hair has possibly mobilized a preformed thrombus from the vertebral aneurysm or stenosis into the basilar artery, leading to the ischemic lesions in both hippocampi, which are usually part of the posterior cerebral artery supply territory [13]. If DWI lesions are found in TGA patients, they are punctiform and either in one or both hippocampi, but rarely more than one per side [4-7]; in contrast, we found bilateral punctiform lesions as well as an additional patchy diffusion lesion in the right hippocampus (Fig. 2). Isolated hippocampal infarcts, although usually larger than in our patient, commonly present with dizziness, confusion, and cognitive and memory deficits, are mainly of embolic nature and in one-third of patients due to vertebrobasilar large-artery disease [11]. Thus, we believe that the diffusion restrictions in our case are, in fact, ischemic lesions from basilar artery embolism caused by the vertebral artery 

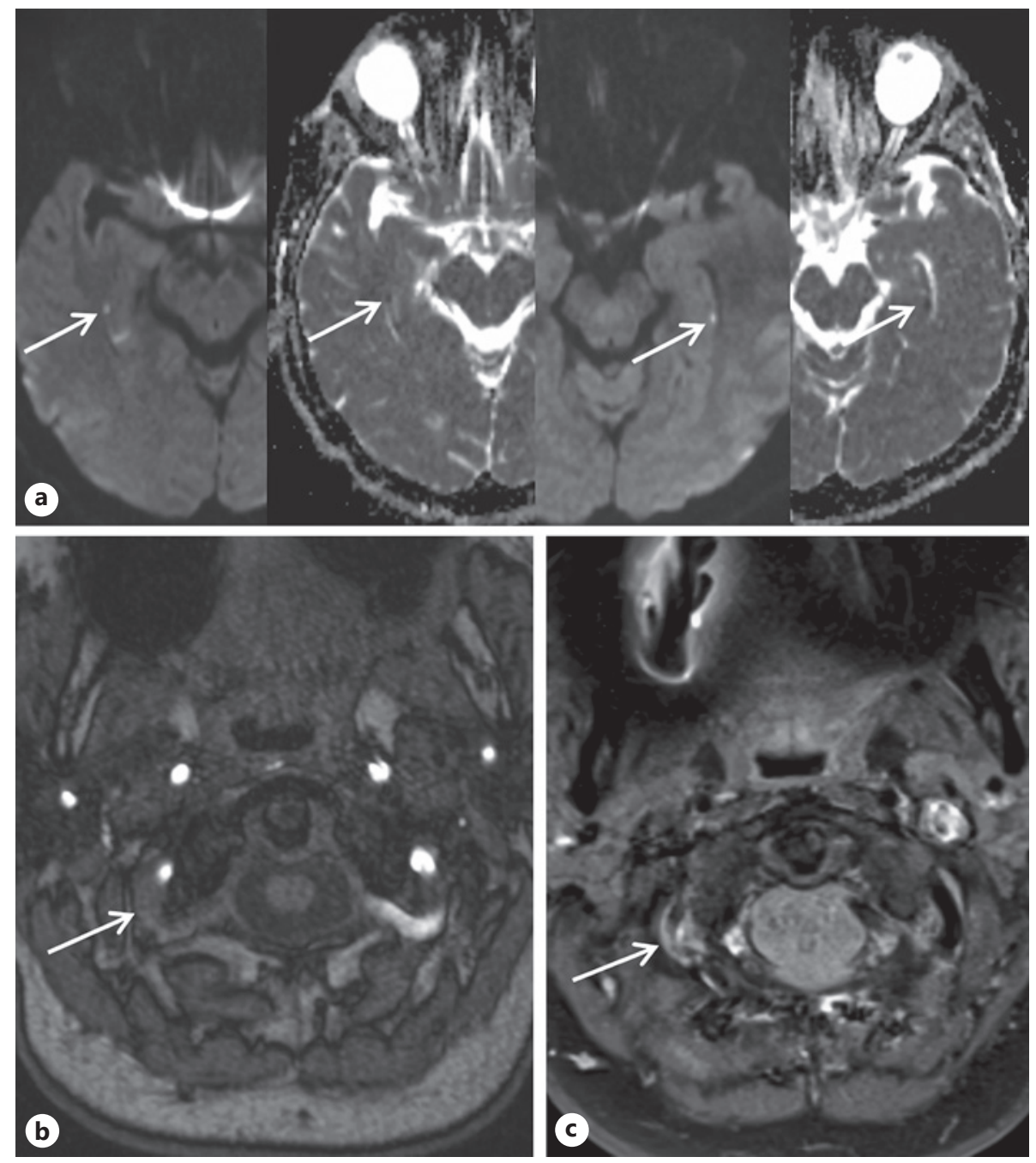

Fig. 2. Sixty-one-year-old female patient with clinical presentation of TGA due to basilar artery embolism from VA dissection, performed $25 \mathrm{~h}$ after onset on a $1.5 \mathrm{~T}$ MRI. a Axial DWI and ADC images showing bilateral small punctate region of abnormally restricted diffusion on DWI/ADC sequences in the CA1 area of the hippocampus (arrow) (lateral edge of the hippocampal gyrus abutting the temporal horn). b Axial TOF MRA reveals occlusion of the right VA (arrow) in comparison to the normally patent left VA. c Axial $P D$-weighted images with fat saturation showing an extensive mural hematoma of the right VA (arrow) with an occlusion of the vessel lumen indicating right VA dissection. PD, proton density; ADC, apparent diffusion coefficient; TOF, time-of-flight; VA, vertebral artery.

dissection. However, the clinical presentation showed overlap with TGA and alternative causes for the accompanying symptoms can be discussed: general weakness and dizziness are unspecific symptoms and can occur in patients with TGA [3] or could have been caused by benign paroxysmal positional vertigo induced by head maneuvering, the hemiataxia was only subtle and could have been within the normal range, and the rapid return of memory function within $24 \mathrm{~h}$ is typical for TGA. Manipulation on the head and neck, despite not being a typical trigger for TGA, could have led to venous congestion, which has been linked to TGA [14], especially since our patient had history of venous insufficiency. In summary, our case shows that TGA can be a symptom of ischemic lesions in the hippocampus. Especially, cases with presentation of additional focal neurologic symptoms or atypical distribution or 
appearance of the DWI (multiple/patchy) lesions should be treated as transient basilar embolism, and therefore, lead to routine workup of ischemic stroke and subsequent administration of secondary prevention.

\section{Acknowledgments}

We thank Dr. Moritz von Cranach for providing the duplex sonography image of the vertebral artery dissection (Fig. 1).

\section{Statement of Ethics}

The paper is exempt from Ethical Committee approval due to the regulations of the Ethical Committee of our institution regarding case reports. Written informed consent was obtained from the patient for publication of this case report and any accompanying images.

\section{Conflict of Interest Statement}

The authors have no conflicts of interest to declare.

\section{Funding Sources}

The authors did not receive support from any organization for the submitted work.

\section{Author Contributions}

E.M., D.K., C.S., and W.-D.N. treated the patient. T.A. and C.T. acquired and analyzed image data. E.M. and D.K. drafted the manuscript. W.-D.N., C.S., T.A., and C.T. revised the manuscript. All authors read and approved the final version of the manuscript.

\section{Data Availability Statement}

The data that support the findings of this study are not publicly available because of the possibility of face reconstruction/recognition from the MRI/CT scans but are available from the corresponding author E.M. (ernst.mayerhofer@uniklinik-freiburg.de) upon reasonable request.

\section{References}

1 Hodges JR, Warlow CP. Syndromes of transient amnesia: towards a classification. A study of 153 cases. J Neurol Neurosurg Psychiatry. 1990;53(10):834-43.

2 Quinette P, Guillery-Girard B, Dayan J, de la Sayette V, Marquis S, Viader F, et al. What does transient global amnesia really mean? Review of the literature and thorough study of 142 cases. Brain. 2006;129(Pt 7):1640-58.

3 Arena JE, Rabinstein AA. Transient global amnesia. Mayo Clin Proc. 2015;90(2):264-72.

4 Strupp M, Brüning R, Wu RH, Deimling M, Reiser M, Brandt T. Diffusion-weighted MRI in transient global amnesia: elevated signal intensity in the left mesial temporal lobe in 7 of 10 patients. Ann Neurol. 1998;43(2):164-70.

\section{Karger'}


5 Sedlaczek O, Hirsch JG, Grips E, Peters CN, Gass A, Wohrle J, et al. Detection of delayed focal MR changes in the lateral hippocampus in transient global amnesia. Neurology. 2004;62(12):2165-70.

6 Yang Y, Kim S, Kim JH. Ischemic evidence of transient global amnesia: location of the lesion in the hippocampus. J Clin Neurol. 2008;4(2):59-66.

7 Winbeck K, Etgen T, von Einsiedel HG, Röttinger M, Sander D. DWI in transient global amnesia and TIA: proposal for an ischaemic origin of TGA. J Neurol Neurosurg Psychiatry. 2005;76(3):438-41.

8 Huber R, Aschoff AJ, Ludolph AC, Riepe MW. Transient global amnesia. Evidence against vascular ischemic etiology from diffusion weighted imaging. J Neurol. 2002;249(11):1520-4.

9 Gass A, Gaa J, Hirsch J, Schwartz A, Hennerici MG. Lack of evidence of acute ischemic tissue change in transient global amnesia on single-shot echo-planar diffusion-weighted MRI. Stroke. 1999;30(10):2070-2.

10 Woolfenden AR, O'Brien MW, Schwartzberg RE, Norbash AM, Tong DC. Diffusion-weighted MRI in transient global amnesia precipitated by cerebral angiography. Stroke. 1997;28(11):2311-4.

11 Kumral E, Deveci EE, Erdoğan C, Enüstün C. Isolated hippocampal infarcts: vascular and neuropsychological findings. J Neurol Sci. 2015;356(1-2):83-9.

12 Mangla A, Navi BB, Layton K, Kamel H. Transient global amnesia and the risk of ischemic stroke. Stroke. 2014; 45(2):389-93.

13 Erdem A, Yaşargil G, Roth P. Microsurgical anatomy of the hippocampal arteries. J Neurosurg. 1993;79(2): 256-65.

14 Han K, Chao AC, Chang FC, Chung CP, Hsu HY, Sheng WY, et al. Obstruction of venous drainage linked to transient global amnesia. PLoS One. 2015;10(7):e0132893. 